\title{
MIMO radar waveform design with peak and sum power constraints
}

Merline Arulraj ${ }^{1 *}$ and Thiruvengadam S Jeyaraman ${ }^{2}$

\begin{abstract}
Optimal power allocation for multiple-input multiple-output radar waveform design subject to combined peak and sum power constraints using two different criteria is addressed in this paper. The first one is by maximizing the mutual information between the random target impulse response and the reflected waveforms, and the second one is by minimizing the mean square error in estimating the target impulse response. It is assumed that the radar transmitter has knowledge of the target's second-order statistics. Conventionally, the power is allocated to transmit antennas based on the sum power constraint at the transmitter. However, the wide power variations across the transmit antenna pose a severe constraint on the dynamic range and peak power of the power amplifier at each antenna. In practice, each antenna has the same absolute peak power limitation. So it is desirable to consider the peak power constraint on the transmit antennas. A generalized constraint that jointly meets both the peak power constraint and the average sum power constraint to bound the dynamic range of the power amplifier at each transmit antenna is proposed recently. The optimal power allocation using the concept of waterfilling, based on the sum power constraint, is the special case of $p=1$. The optimal solution for maximizing the mutual information and minimizing the mean square error is obtained through the Karush-Kuhn-Tucker (KKT) approach, and the numerical solutions are found through a nested Newton-type algorithm. The simulation results show that the detection performance of the system with both sum and peak power constraints gives better detection performance than considering only the sum power constraint at low signal-to-noise ratio.
\end{abstract}

\section{Introduction}

Multiple-input multiple-output (MIMO) radar is an emerging technology that has significant potential for advancing the state of the art of modern radar. The application of information theory to radar was proposed more than 50 years ago by Woodward and Davies [1,2]. In [3], maximizing the mutual information (MI) between a Gaussian-distributed extended target reflection and the received signal was suggested. This is believed to be the first to apply information theory to radar waveform design. An information theoretic approach is used in [4] to design radar waveforms suitable for simultaneously estimating and tracking the parameters of multiple targets. The authors in [5] have introduced a criterion for waveform selection in adaptive radar and other sensing applications, which are also based on information theory.
There exist some recent works in the area of radar target identification and classification, which apply both information theoretic and estimation theoretic criteria for optimal waveform design. For example, the research in [6] considered waveform design for MIMO radar (e.g., see [7-15]) by optimizing two criteria: maximization of the MI and minimization of the minimum mean square error (MMSE). It was demonstrated that these two different criteria yield essentially the same optimum solution. Further, this is also true for an asymptotic formulation [6], which requires only the knowledge of power spectral density (PSD). However, it might be very difficult to obtain perfect knowledge of the PSD in practice. In such a circumstance, robust procedures, which can overcome those problems by incorporating a modeling uncertainty into the design from the outset [16],

\footnotetext{
* Correspondence: a_merline@yahoo.co.in

'Department of Electronics and Communication Engineering, Sethu Institute of Technology, Kariapatti, Virudhunagar District 626 115, India

Full list of author information is available at the end of the article
} 
seem quite attractive. For the design of optimal signal for the estimation of correlated MIMO channels, the information theoretic and estimation theoretic criteria are used in [17].

Naghibi and Behnia [18] have investigated the problem of waveform design for target classification and estimation in the presence of clutter using a MMSE estimator for widely separated and closely spaced antenna configurations. It is shown that the waveform design that resulted from MI, MMSE, and normalized mean square error is all different when the noise is assumed to be colored and when the target and noise statistics are not perfectly known [19]. We have proposed a waveform design technique for minimizing the mean square error for estimating the target impulse response in [20], and it has not been addressed before.

The optimal solution for waveform design employs waterfilling that uses sum power constraint (SPC) at the transmitter to allocate limited power appropriately [6]. However, it results in wide power variations across the transmit antennas, and it poses a severe constraint on the dynamic range and peak power amplifier at each antenna. In a multi-antenna system where each antenna has the same power amplifier, this would result in peak power clipping. Recently, a transmit beamformer design is proposed under the uniform elemental power constraint. It has been shown that transmit beamforming with the uniform elemental power constraint has better bit error rate performance compared to transmit beamforming with peak power clipping [21]. Another simple way to control the dynamic range of the power amplifier at each transmit antenna is by imposing the per-antenna power constraint such that the maximum eigenvalue of the channel power matrix is less than the specified per-antenna power [22]. In a multi-antenna base station where each antenna has its own power amplifier in its analog front-end and is limited individually by the linearity of the power amplifier, a power constraint imposed on a per-antenna basis is more realistic [23]. The focus of this paper is to design a beamforming vector that minimizes the per-antenna power on each transmit antenna while enforcing a set of SINR constraints on each user.

Recently, the $p$-norm constraint which jointly meets the sum power constraint and the maximum average individual power constraint has been proposed [24]. From a mathematical point of view, the sum power constraint turns out to be the case of a family of constraints with $p=1$, and the equal power constraint turns out as $p=\infty$. Therefore, the $p$-norm power constraint seems to be a very powerful measurement to characterize a more general constraint for MIMO system. The directional derivative method is shown to be an efficient method to solve the optimum linear transceivers, subject to the $p$-norm constraint $[24,25]$. In [25], the mutual information between the input and output of Gaussian vector channels is considered, given the channel state information.

This paper addresses the problem of designing waveforms for MIMO radar that maximizes mutual information and that minimizes the mean square error in estimating the target impulse response subject to the $p$ norm constraint assuming that the radar transmitter has knowledge of the target PSD. The target PSD could be obtained through some feedback mechanism referred to as covariance feedback. The focus of this paper is to meet the peak power constraint and the sum power constraint to a maximum possible extent. The rest of this paper is organized as follows: In Section 2, the signal model is presented. The general concept of $p$-norm is introduced in Section 3. The problem formulation is briefed in Section 4. The waveform design with the $p$ norm constraint using the Karush-Kuhn-Tucker (KKT) approach is derived in Section 5. Detection performance of the MIMO radar waveform is considered in Section 6, and a numerical example is given in Section 7. Section 8 concludes this paper.

\subsection{Notation}

Bold uppercase and lowercase letters denote matrices and vectors, respectively. Superscripts $\{.\}^{H}$ and $\{.\}^{T}$ are used to denote the complex conjugate transpose and transpose of a matrix, respectively. $\operatorname{det}\{$.$\} and \operatorname{tr}\{$.$\} repre-$ sent the determinant and trace of a matrix, respectively. The symbol " $\|\bullet\|$ " denotes the Euclidean norm of a vector, and $\operatorname{diag}\{\boldsymbol{a}\}$ denotes a diagonal matrix with its diagonal given by the vector a. Complex Gaussian distribution with mean $m$ and covariance matrix $\boldsymbol{R}$ is denoted by $\mathcal{N}(m, \boldsymbol{R})$. Finally, $(a)^{+}$denotes the positive part of $a$, i.e., $(a)^{+}=\max [0, a]$.

\section{System model}

Consider a MIMO radar equipped with $M$ transmitting antenna elements and $N$ receiving antenna elements with extended target. The target is assumed to be pointlike between each pair of transmit and receive antennas. The received signal component at the $n$th antenna element in the $k$ th time instant is expressed as

$$
y_{n}(k)=\sum_{i=1}^{M} h_{i n} s_{i}(k)+\xi_{n}(k), \quad k=1, \ldots, K,
$$

where $s_{i}(k)$ represents the transmit signal at the $i$ th transmit antenna, $h_{i n}$ is the target impulse response from the $i$ th transmit antenna to the $n$th receive antenna, and $\xi_{n}(k)$ is the noise in the $n$th receive antenna. The components of the noise vector are assumed to be independent 
and identically distributed (i.i.d.) Gaussian random variables with zero mean and variance $\sigma_{\xi}^{2}$. In vector form, the signal model is written as

$$
y_{n}(k)=\boldsymbol{h}_{n}{ }^{T} \boldsymbol{s}(k)+\xi_{n}(k),
$$

where $\boldsymbol{h}_{n}=\left[h_{1 n}, h_{2 n}, \ldots, h_{M n}\right]^{T}$ and $\boldsymbol{s}(k)=\left[s_{1}(k), s_{2}(k), \ldots\right.$, $\left.s_{M}(k)\right]^{T}$. The received signal at the $n$th receive element obtained by stacking the $K$ samples for an observation time of $T$ seconds in a row is given by

$$
\boldsymbol{y}_{n}=\boldsymbol{h}_{n}{ }^{T} \boldsymbol{S}^{T}+\boldsymbol{\xi}_{n}
$$

where $\boldsymbol{S}=[\boldsymbol{s}(1), \boldsymbol{s}(2), \ldots, \boldsymbol{s}(K)]^{\boldsymbol{T}}$. It is assumed that the channel is unchanged during the observation time of $T$ seconds. Collecting the received waveforms from all the $N$ receive elements, the received signal in matrix form can be written as

$$
\boldsymbol{Y}=\boldsymbol{S H}+\boldsymbol{\xi},
$$

where $\boldsymbol{Y}=\left[\boldsymbol{y}_{1}^{T}, \boldsymbol{y}_{2}^{T}, \ldots, \boldsymbol{y}_{N}^{T}\right]$ is Gaussian distributed with zero mean and covariance $\left(\boldsymbol{S} \boldsymbol{R}_{H} \boldsymbol{S}^{H}+\sigma_{\xi}^{2} \boldsymbol{I}_{k}\right)$, the columns of $\boldsymbol{H}=\left[\boldsymbol{h}_{1}, \boldsymbol{h}_{2}, \ldots, \boldsymbol{h}_{N}\right]$ are i.i.d. with distribution $\mathcal{N}\left(0, \boldsymbol{R}_{H}\right)$ and the columns of $\xi=\left[\xi_{1}^{T}, \xi_{2}^{T}, \ldots, \xi_{N}^{T}\right]$ are i.i.d. with zero mean and covariance matrix $\sigma_{\xi}^{2} \boldsymbol{I}_{K}$.

\section{Preliminaries}

The concept of $p$-norm and its relation to various power constraints is briefly summarized in this section. In linear algebra theory, the $p$-norm is given by

$$
\|x\|_{p}:=\left(\sum_{i=1}^{n}\left|x_{i}\right|^{p}\right)^{1 / p} \text { for } p \geq 1
$$

1. For $p=1,\|x\|_{1}:=\sum_{i=1}^{n}\left|x_{i}\right|$. This is 1 -norm and it is simply the sum of the absolute values of $x_{i}$. So this refers to the sum power constraint if $x_{i}$ denotes the power in each antenna.

2. For $p=\infty,\|x\|_{\infty}:=\max \left(\left|x_{1}, \ldots,\right| x_{M}||\right)$. In linear algebra theory, this infinity norm is a special case of the uniform norm. So this refers to equal power allocation.

3. For $1<p<\infty$, the $p$-norm constraint can be formulated into an optimization problem and can satisfy both the sum power constraint with an upper bound $\beta$ that is not tight and a peak power constraint with threshold $\alpha$ for an appropriate value for ' $p$ ' as discussed below.

\section{Problem formulation}

In this paper, the design of MIMO radar waveform with combined peak and sum power constraints is addressed using two different criteria. The first one is to maximize the conditional mutual information between the target impulse response and the reflected waveform. The second one is to minimize the mean square error in estimating the target impulse response.

\subsection{MI criterion}

The conditional mutual information between the received signal $\boldsymbol{Y}$ and the target impulse response $\boldsymbol{H}$, given the knowledge of $S$ is

$$
\boldsymbol{I}(\boldsymbol{Y} ; \boldsymbol{H} / \boldsymbol{S})=h(\boldsymbol{Y} / \boldsymbol{S})-h(\boldsymbol{Y} / \boldsymbol{H}, \boldsymbol{S}) .
$$

From [26], $h(\boldsymbol{Y} / \boldsymbol{S})=\log \left[\operatorname{det}\left(\boldsymbol{S} \boldsymbol{R}_{H} \boldsymbol{S}^{H}+\sigma_{\xi}^{2} \boldsymbol{I}_{k}\right)\right]$ and $h(\boldsymbol{Y} / \boldsymbol{H}, \boldsymbol{S})=$ $h(\xi)$; then, the mutual information in (5) can be written as

$$
\boldsymbol{I}(\boldsymbol{Y} ; \boldsymbol{H} / \boldsymbol{S})=\log \left[\operatorname{det}\left(\boldsymbol{I}_{k}+\boldsymbol{\sigma}_{\xi}^{-2} \boldsymbol{S} \boldsymbol{R}_{H} \boldsymbol{S}^{H}\right)\right] .
$$

Using the determinant property, $\operatorname{det}\left(\boldsymbol{I}_{p}+\boldsymbol{A} \boldsymbol{B}\right)=\operatorname{det}\left(\boldsymbol{I}_{q}+\right.$ $\boldsymbol{B A})$, (6) can be written as

$$
\boldsymbol{I}(\boldsymbol{Y} ; \boldsymbol{H} / \boldsymbol{S})=\log \left[\operatorname{det}\left(\boldsymbol{I}_{M}+\sigma_{\xi}^{-2} \boldsymbol{R}_{H} \boldsymbol{S}^{H} \boldsymbol{S}\right)\right] .
$$

If the variance of noise is assumed to be unity,

$$
\boldsymbol{I}(\boldsymbol{Y} ; \boldsymbol{H} / \boldsymbol{S})=\log \left[\operatorname{det}\left(\boldsymbol{I}_{M}+\boldsymbol{R}_{H} \boldsymbol{S}^{H} \boldsymbol{S}\right)\right]
$$

The objective is to maximize the mutual information $\boldsymbol{I}(\boldsymbol{Y} ; \boldsymbol{H} / \boldsymbol{S})$. In the case of the sum power constraint, the constraint is given by

$$
\left[\operatorname{tr}\left(\boldsymbol{S}^{H} \boldsymbol{S}\right)\right] \leq \beta
$$

where $\beta$ is the sum of the average transmit powers. However, it results in wide power variations across the transmit antennas [22]. So the $p$-norm power constraint that jointly satisfies the sum power constraint and the peak power constraint is considered here [25]. Then, the problem of waveform design can be expressed as

$$
\begin{aligned}
& \max _{\boldsymbol{s}} \log \left[\operatorname{det}\left(\boldsymbol{I}_{M}+\boldsymbol{R}_{H} \boldsymbol{S}^{H} \boldsymbol{S}\right)\right] \\
& \text { s.t. }\left[\operatorname{tr}\left(\boldsymbol{S}^{H} \boldsymbol{S}\right)^{p}\right]^{1 / p} \leq J,
\end{aligned}
$$

where $J$ is a constant, the value of which depends on the constraint. Let $\alpha$ be the constraint on the peak power on the antenna. If $p=1$, the constraint is $\operatorname{tr}\left(\boldsymbol{S}^{H} \boldsymbol{S}\right)=J$, then the constant $J$ will be equal to the sum power constraint $\beta$. If $p=\infty$, the norm becomes aninfinity norm which is a special case of the uniform norm in linear algebra theory. To meet both the sum power constraint and the peak power constraint, the maximum value cannot be greater than the equal power, i.e., $\beta / M$. For values of $p$ within the interval $1<p<\infty, J=\alpha$ satisfies both the per-antenna power 
constraint and sum power constraint. The individual power constraint $\alpha$ can be chosen in the interval $\left[\frac{\beta}{M}, \beta\right]$. The norm factor $p$ is appropriately chosen as [22]

$$
p=\frac{\ln M}{\ln (\alpha M / \beta)}
$$

Applying singular value decomposition on $\boldsymbol{R}_{H}$, it can be expressed as $\boldsymbol{R}_{H}=\boldsymbol{U} \boldsymbol{\Lambda} \boldsymbol{U}^{H}$, where $\boldsymbol{\Lambda}=\operatorname{diag}\left(\lambda_{11}, \lambda_{22}, \ldots\right.$, $\lambda_{M M}$ ), where $\lambda_{i i}$ is the eigenvalue of the covariance matrix of the target impulse response. Substituting for $\boldsymbol{R}_{H}$ in (7) and simplifying,

$$
\boldsymbol{I}(\boldsymbol{Y} ; \boldsymbol{H} / \boldsymbol{S})=\log \left[\operatorname{det}\left(\boldsymbol{I}_{M}+\Lambda \boldsymbol{X}^{H} \boldsymbol{X}\right)\right]
$$

where $\boldsymbol{X}=\boldsymbol{S} \boldsymbol{U}$ is a $(K \times M)$ matrix. Let $\boldsymbol{D}=\boldsymbol{X}^{\boldsymbol{H}} \boldsymbol{X}$. Since $\boldsymbol{U}$ is unitary, $\operatorname{tr}\left(\boldsymbol{S}^{H} \boldsymbol{S}\right)=\operatorname{tr}\left(\boldsymbol{X}^{H} \boldsymbol{X}\right)$. Now the problem formulation can be expressed as

$$
\begin{gathered}
\max _{\boldsymbol{D}} \log \left[\operatorname{det}\left(\boldsymbol{I}_{M}+\Lambda \mathbf{D}\right)\right] \\
\text { s.t. }\left[\operatorname{tr}(\boldsymbol{D})^{p}\right]^{(1 / p)} \leq J .
\end{gathered}
$$

\subsection{MMSE criterion}

The problem of radar waveform design under the scenario of target identification requires the estimation of target impulse response. MMSE, in estimating the target impulse response, is given by [3]

$$
\text { MMSE }=\operatorname{tr}\left\{\left(\sigma_{\xi}^{-2} \boldsymbol{S}^{H} \boldsymbol{S}+\boldsymbol{R}_{H}^{-1}\right)^{-1}\right\}
$$

If the noise is assumed to have unit variance, then

$$
\text { MMSE }=\operatorname{tr}\left\{\left(\boldsymbol{S}^{H} \boldsymbol{S}+\boldsymbol{R}_{H}{ }^{-1}\right)^{-1}\right\} .
$$

As given for mutual information, the problem formulation for MMSE could be given as

$$
\begin{gathered}
\min _{\boldsymbol{D}} \operatorname{tr}\left\{\left(\boldsymbol{D}+\boldsymbol{\Lambda}^{-1}\right)^{-1}\right\} \\
\text { s.t. }\left[\operatorname{tr}(\boldsymbol{D})^{p}\right]^{(1 / p)} \leq J .
\end{gathered}
$$

\section{Waveform design with $p$-norm power constraint}

According to Hadamard's inequality, the optimal solution of (11) and (14) can be achieved when $\left(I_{M}+\Lambda \mathrm{D}\right)$ in
(11) and $\left(\mathbf{D}+\Lambda^{-1}\right)^{-1}$ in (14) are diagonal. Hadamard's inequalities for the determinant and trace of an $n \times n$ positive semidefinite Hermitian matrix $\boldsymbol{A}$ are

$$
\begin{aligned}
& \operatorname{det}(\boldsymbol{A}) \leq \prod_{i=1}^{n} a_{i i}, \\
& \operatorname{tr}\left(\boldsymbol{A}^{-1}\right) \geq \sum_{i=1}^{n} \frac{1}{a_{i i}},
\end{aligned}
$$

where $a_{i i}$ is the $i$ th diagonal element of $\boldsymbol{A}$, and equality is achieved in both cases if and only if $\boldsymbol{A}$ is diagonal [27]. Thus, $\mathbf{D}=\boldsymbol{X}^{H} \boldsymbol{X}$ must be a diagonal matrix with nonnegative elements $d_{i i} \geq 0, \forall i \in[1, M]$. Now, the mutual information in (10) can be written as

$$
\boldsymbol{I}(\boldsymbol{Y} ; \boldsymbol{H} / \boldsymbol{S})=\log \left[\operatorname{det}\left(\boldsymbol{I}_{M}+\Lambda \mathrm{D}\right)\right]
$$

It can be shown that (16) is concave as a function of $\mathbf{D}$ [28]. Similarly, the minimum mean square error in (13) can be written as

$$
\text { MMSE }=\operatorname{tr}\left\{\left(\boldsymbol{D}+\boldsymbol{\Lambda}^{-1}\right)^{-1}\right\} .
$$

The MMSE function in (17) is convex as a function of $\mathbf{D}$ [18]. If $\mathbf{D}=\boldsymbol{X}^{H} \boldsymbol{X}$ should be a diagonal matrix, the columns of $X$ should be orthogonal. Hence, $X$ is factored as [29]

$$
\boldsymbol{X}=\varphi \mathbf{D}^{1 / 2},
$$

where the columns of $\varphi$ are orthonormal. As $X=S U$, the transmitted signal matrix is given by

$$
\boldsymbol{S}=\varphi \boldsymbol{D}^{(1 / 2)} \boldsymbol{U}^{H},
$$

$$
\boldsymbol{S}=\varphi\left(\operatorname{diag}\left(d_{11}, d_{22}, \ldots, d_{M M}\right)\right)^{(1 / 2)} \boldsymbol{U}^{H},
$$

where $d_{i i}$ is the diagonal element of $\mathbf{D}$. The two problems given in (11) and (14) are convex optimization problems that can be solved using the KKT optimality conditions [28].

\subsection{MI criterion}

The problem statement in (11) is now written as

$$
\begin{aligned}
& \max \sum_{i=1}^{M} \log \left(1+\lambda_{i} d_{i}\right), \\
& \sum_{i=1}^{M} d_{i}^{p} \leq J^{p} \text { and } d_{i} \geq 0 .
\end{aligned}
$$


The Lagrangian for (20) can be written as

$$
\begin{aligned}
L(\boldsymbol{d}, \boldsymbol{\eta}, h)= & \sum_{i=1}^{M} \log \left(1+\lambda_{i} d_{i}\right)+\sum_{i=1}^{M} d_{i} \eta_{i} \\
& +h\left(J^{p}-\sum_{i=1}^{M} d_{i}^{p}\right),
\end{aligned}
$$

where $\boldsymbol{d}=\left(d_{1}, d_{2}, \ldots, d_{M}\right), \boldsymbol{\eta}=\left(\eta_{1}, \eta_{2}, \ldots, \eta_{M}\right)$, and $\boldsymbol{\eta}$ and $h$ are Lagrangian multipliers. The optimality conditions are [20]

$$
\begin{gathered}
\frac{\partial L(\boldsymbol{d}, \boldsymbol{\eta}, h)}{\partial d_{i}}=\frac{\lambda_{i}}{1+\lambda_{i} d_{i}}+\eta_{i}-h p d_{i}^{p-1}=0 \\
d_{i}, \eta_{i} \geq 0, i=1,2, \ldots, M \\
d_{i} \eta_{i}=0, i=1,2, \ldots, M \\
J^{p}-\sum_{i=1}^{M} d_{i}^{p}=0 .
\end{gathered}
$$

If $\lambda_{i}>0,(22)$ can be solved as

$$
\begin{gathered}
\frac{\lambda_{i}}{1+\lambda_{i} d_{i}}=h p d_{i}^{p-1}-\eta_{i} \\
\frac{\lambda_{i} d_{i}^{1-p}}{1+\lambda_{i} d_{i}}=h p-\eta_{i} d_{i}^{1-p} \\
\frac{1+\lambda_{i} d_{i}}{\lambda_{i}} d_{i}^{p-1}=\frac{1}{h p} \\
d_{i}^{p}+\frac{1}{\lambda_{i}} d_{i}^{p-1}=\mu
\end{gathered}
$$

where $\mu=\frac{1}{h p}$. If $\lambda_{i}=0$, then $d_{i}=0$.

\subsubsection{Case 1: sum power constraint $(p=1)$}

The transmit sum power constraint turns out as the special case of the $p$-norm constraint at $p=1$. Substituting $p=1$ in (23) gives

$$
d_{i}=\left(\mu-\frac{1}{\lambda_{\mathrm{i}}}\right)^{+}
$$

$\mu$ such that $\sum_{i=1}^{M} d_{i}=J$.

This case yields the well-known waterfilling solution.

\subsubsection{Case 2:equal power constraint $(p=\infty)$}

The case $p=\infty$ turns out as the equal power constraint of the $p$-norm constraint. It is known that

$$
\|\mathbf{D}\|_{p}=\max \left(\left|d_{1}\right|,\left|d_{2}\right|, \ldots,\left|d_{M}\right|\right)
$$

\subsubsection{Case 3: peak and sum power constraints $(1<p<\infty)$} The solution of $d_{i}$ for $1 \leq i \leq M$ is obtained by solving the simultaneous equations which are obtained using KKT optimality conditions. The simultaneous equation in (23) can be solved using a fast quadratically convergent algorithm for finding numerical solutions. It consists of nested Newton iterations of the general type $x_{n+1}=x_{n}-h\left(x_{n}\right)$ / $h^{\prime}\left(x_{n}\right)$, useful for finding a solution of $x$ of $h(x)=0$.

The monotone function is given by

$$
q_{i}(d)=d^{p}+\frac{1}{\lambda_{i}} d^{p-1}, d \geq 0,1 \leq i \leq M
$$

The zero of the monotone function is

$$
L(\mu)=\sum_{i}\left[q_{i}^{-1}(\mu)\right]^{p}-J^{p}, \mu \geq 0,1 \leq i \leq M .
$$

For $\mu=\mu^{(n)}$, the iteration is

$$
d_{i, k+1}^{(n)}=d_{i, k}^{(n)}-\frac{\left(d_{i, k}^{(n)}\right)^{p}+\left(d_{i, k}^{(n)}\right)^{p-1} / \lambda_{i}-\mu^{(n)}}{p\left(d_{i, k}^{(n)}\right)^{p-1}+(p-1)\left(d_{i, k}^{(n)}\right)^{p-2} / \lambda_{i}}, 1 \leq i \leq M .
$$

The outer iteration is

$$
\mu^{(n+1)}=\mu^{(n)}-\frac{L\left(\mu^{(n)}\right)}{L^{\prime}\left(\mu^{(n)}\right)},
$$

where

$$
\begin{aligned}
L^{\prime}(\mu) & =\sum_{i} p\left[q_{i}^{-1}(\mu)\right]^{p-1} q_{i}^{-1^{\prime}}(\mu) \\
& =\sum_{i} \frac{1}{1+\frac{p-1}{p \lambda_{i}}\left[q_{i}^{-1}(\mu)\right]^{-1}} .
\end{aligned}
$$

\subsection{MMSE criterion}

The problem statement of (14) can be written as

$$
\begin{array}{ll}
\min & \sum_{i=1}^{M}\left(\frac{\lambda_{i}}{d_{i} \lambda_{i}+1}\right) \\
\text { s.t. } & \sum_{i=1}^{M} d_{i}^{p} \leq J^{p} \text { and } d_{i} \geq 0 .
\end{array}
$$

The Lagrangian for (31) can be written as

$$
\begin{aligned}
L(\boldsymbol{d}, \boldsymbol{\eta}, h)= & \sum_{i=1}^{M}\left(\frac{\lambda_{i}}{d_{i} \lambda_{i}+1}\right)+\sum_{i=1}^{M} d_{i} \eta_{i} \\
& +h\left(J^{p}-\sum_{i=1}^{M} d_{i}^{p}\right)
\end{aligned}
$$

where $\boldsymbol{d}=\left(d_{1}, d_{2}, \ldots, d_{M}\right), \boldsymbol{\eta}=\left(\eta_{1}, \eta_{2}, \ldots, \eta_{M}\right)$, and $\boldsymbol{\eta}$ and $h$ are Lagrangian multipliers. The optimality conditions are 


$$
\begin{gathered}
\frac{\partial L(\boldsymbol{d}, \boldsymbol{\eta}, h)}{\partial d_{i}}=\frac{-\lambda_{i}^{2}}{\left(1+\lambda_{i} d_{i}\right)^{2}}+\eta_{i}-h p d_{i}^{p-1}=0 \\
d_{i}, \eta_{i} \geq 0, \quad i=1,2, \ldots, M \\
d_{i} \eta_{i}=0, \quad i=1,2, \ldots, M \\
J^{p}-\sum_{i=1}^{M} d_{i}^{p}=0 .
\end{gathered}
$$

If $\lambda_{i}>0$, (25) can be solved as

$$
\begin{gathered}
\frac{-\lambda_{i}^{2}}{\left(1+\lambda_{i} d_{i}\right)^{2}}=h p d_{i}^{p-1}-\eta_{i} \\
d_{i}^{p+1}+\frac{2}{\lambda_{i}} d_{i}^{p}+\frac{1}{\lambda_{i}{ }^{2}} d_{i}^{p-1}=\mu .
\end{gathered}
$$

\subsubsection{Case 1: sum power constraint $(p=1)$}

Substituting $p=1$ in (34) gives the well-known waterfilling solution as in the case of the MI criterion:

$$
\begin{gathered}
\qquad d_{i}=\left(\mu-\frac{1}{\lambda_{\mathrm{i}}}\right)^{+} \\
\mu \text { such that } \sum_{i=1}^{M} d_{i}=J .
\end{gathered}
$$

\subsubsection{Case 2:equal power constraint $(p=\infty)$}

For $p=\infty$,

$$
\|\mathbf{D}\|_{p}=\max \left(\left|d_{1}\right|,\left|d_{2}\right|, \ldots,\left|d_{M}\right|\right) .
$$

\subsubsection{Case 3: peak and sum power constraints $(1<p<\infty)$}

For the case of peak and sum power constraints, (34) can be solved using the nested Newton algorithm. The monotone function is given by

$$
q_{i}(d)=d^{P+1}+\frac{2}{\lambda_{\mathrm{i}}} d^{P}+\frac{1}{\lambda_{\mathrm{i}}^{2}} d^{P-1}, d \geq 0,1 \leq i \leq M .
$$

The update equation for $d_{i}$ is

$$
d_{i, k+1}^{(n)}=d_{i, k}^{(n)}-\frac{\frac{\left(d_{i, k}^{(n)}\right)^{p-1}}{\lambda_{\mathrm{i}}{ }^{2}}+\frac{2\left(d_{i, k}^{(n)}\right)^{p}}{\lambda_{\mathrm{i}}}+\left(d_{i, k}^{(n)}\right)^{p+1}-\mu^{(n)}}{(p-1) \frac{\left(d_{i, k}^{(n)}\right)^{p-2}}{\lambda_{\mathrm{i}}{ }^{2}}+\frac{2 p\left(d_{i, k}^{(n)}\right)^{p-1}}{\lambda_{\mathrm{i}}}+(p+1)\left(d_{i, k}^{(n)}\right)^{p}},
$$$$
1 \leq i \leq M \text {. }
$$

As an initial value in the iteration of (28) and (37), $d_{i, 0}^{(n)}=$ $q_{i}^{-1}\left(\mu_{n-1}\right)$ is chosen, and this yields excellent convergence results.

\section{Detection performance - Neyman-Pearson detector}

The MIMO radar detection problem can be formulated as a binary hypothesis test as

$$
\begin{aligned}
& \mathcal{H}_{0}: \quad \boldsymbol{Y}=\boldsymbol{\xi}, \quad \text { no target, } \\
& \mathcal{H}_{1}: \quad \boldsymbol{Y}=\boldsymbol{S} \boldsymbol{H}+\boldsymbol{\xi}, \quad \text { target exists. }
\end{aligned}
$$

The probability density functions (pdfs) of $\boldsymbol{Y}$ under $\mathcal{H}_{0}$ and $\mathcal{H}_{1}$ are given by [30]

$$
\begin{aligned}
p_{o}(\boldsymbol{Y})= & \frac{1}{\pi^{K N} \operatorname{det}^{N}\left(\sigma_{\xi}^{2} \boldsymbol{I}_{K}\right)} \exp \left\{-\operatorname{tr}\left[\left(\sigma_{\xi}^{-2} \boldsymbol{I}_{K}\right) \boldsymbol{Y} \boldsymbol{Y}^{H}\right]\right\} \\
p_{1}(\boldsymbol{Y})= & \frac{1}{\pi^{K N} \operatorname{det}^{N}\left(\boldsymbol{S} \boldsymbol{R}_{H} \boldsymbol{S}^{H}+\sigma_{\xi}^{2} \boldsymbol{I}_{k}\right)} \\
& \times \exp \left\{-\operatorname{tr}\left[\left(\boldsymbol{S} \boldsymbol{R}_{H} \boldsymbol{S}^{H}+\sigma_{\xi}^{2} \boldsymbol{I}_{k}\right)^{-1} \boldsymbol{Y} \boldsymbol{Y}^{H}\right]\right\},
\end{aligned}
$$

respectively. The log-likelihood becomes

$$
\begin{aligned}
l(Y) & =\log \log \frac{p_{1}(Y)}{p_{o}(Y)} \\
& =\sum_{k=1}^{N} \boldsymbol{y}_{k}^{*}\left[\sigma_{\xi}^{-2} \boldsymbol{I}_{K}-\left(\boldsymbol{S} \boldsymbol{R}_{H} \boldsymbol{S}^{H}+\sigma_{\xi}^{2} \boldsymbol{I}_{k}\right)^{-1}\right] \boldsymbol{y}_{k}^{T}+c_{l}
\end{aligned}
$$

where

$$
c_{l}=N\left[\log \operatorname{det}\left(\sigma_{\xi}^{2} \boldsymbol{I}_{K}\right)-\log \operatorname{det}\left(\boldsymbol{S} \boldsymbol{R}_{H} \boldsymbol{S}^{H}+\sigma_{\xi}^{2} \boldsymbol{I}_{k}\right)\right]
$$

is a constant term independent of $\boldsymbol{Y}$. The optimal Neyman-Pearson detection statistics is given by

$$
T(\boldsymbol{Y})=\sum_{k=1}^{N} \boldsymbol{y}_{k}^{*}\left[\sigma_{\xi}^{-2} \boldsymbol{I}_{K}-\left(\boldsymbol{S} \boldsymbol{R}_{H} \boldsymbol{S}^{H}+\sigma_{\xi}^{2} \boldsymbol{I}_{k}\right)^{-1}\right] \boldsymbol{y}_{k}^{T} .
$$

If $T(\boldsymbol{Y})$ exceeds a given threshold, a target exists. To find the detection threshold, we have

$$
\boldsymbol{y}_{k}^{T} \sim\left\{\begin{array}{ll}
\mathrm{CN}\left(0, \sigma_{\boldsymbol{\xi}}^{2} \boldsymbol{I}_{K}\right), & \mathcal{H}_{0} \\
\operatorname{CN}\left(0, \boldsymbol{S} \boldsymbol{R}_{H} \boldsymbol{S}^{H}+\sigma_{\xi}^{2} \boldsymbol{I}_{k}\right), & \mathcal{H}_{1}
\end{array} .\right.
$$

Let

$$
\mathbf{P}=\sigma_{\xi}^{-2} \boldsymbol{I}_{K^{-}}\left(\boldsymbol{S} \boldsymbol{R}_{H} \boldsymbol{S}^{H}+\sigma_{\xi}^{2} \boldsymbol{I}_{k}\right)^{-1}
$$


Then, we have [31]

$$
2 \boldsymbol{y}_{k}^{*} \mathbf{P} \boldsymbol{y}_{k}^{T} \sim \sum_{j=1}^{K} \alpha_{j}^{(i)} \chi_{2}^{2}(j) \text {. }
$$

Under $\mathcal{H}_{i}, i=0,1$, where $\alpha_{j}^{(i)}$ is the $j$ th eigenvalue of $\mathbf{P}^{1 / 2}\left(\gamma \boldsymbol{S} \boldsymbol{R}_{H} \boldsymbol{S}^{H}+\sigma_{\xi}^{2} \boldsymbol{I}_{k}\right) \mathbf{P}^{1 / 2}, \gamma=0$ under $\mathcal{H}_{0}$ and $\gamma=1$ under $\mathcal{H}_{1}$. Therefore, we have

$$
2 T(\boldsymbol{Y})=2 \sum_{k=1}^{N} \boldsymbol{y}_{k}^{*} \mathbf{P} \boldsymbol{y}_{k}^{T} \sim \sum_{k=1}^{K} \alpha_{k}^{(i)} \chi_{2 N}^{2}(k)
$$

under $\mathcal{H}_{i}, i=0,1$. The test statistics is the weighted sum of chi-squares. It is approximated as gamma distribution [32]. If $C_{q}$ are real positive constants and $N_{q}$ are independent standard normal random variables, $\forall q=$ $1, \ldots, K$, then the pdf of the gamma approximation of $R=\sum_{q=1}^{K} C_{q} N_{q}^{2}$ is given as

$$
f_{R}(r, a, b)=\frac{r^{a-1} e^{-\frac{r}{b}}}{b^{a} \Gamma(a)}
$$

where the parameters $a$ and $b$ are given as

$$
\begin{aligned}
& a=\frac{1}{2}\left[\frac{\left(\sum_{q=1}^{K} C_{q}\right)^{2}}{\sum_{q=1}^{K} C_{q}^{2}}\right], \\
& b=\left[\frac{1}{2}\left(\frac{\sum_{q=1}^{K} C_{q}}{\sum_{q=1}^{K} C_{q}^{2}}\right)\right]^{-1},
\end{aligned}
$$

where $\Gamma$ is the gamma function defined as

$$
\Gamma(a)=\int_{0}^{\infty} t^{a-1} e^{-t} d t
$$

For the test statistics in (43), $C_{q}$ corresponds to $\alpha_{k}^{(i)}$ and $N_{q}^{2}$ corresponds to $\chi_{2 N}^{2}(k) \chi$. After approximating the pdf using the gamma density, the probability of detection $\left(P_{\mathrm{D}}\right)$ and the probability of false alarm $\left(P_{\mathrm{FA}}\right)$ are defined as

$$
\begin{aligned}
P_{\mathrm{D}} & =\int_{\gamma}^{\infty} t^{a_{H 1}-1} \frac{e^{-\frac{t}{b_{H 1}}}}{b_{H 1}^{a_{H 1}} \Gamma\left(a_{H 1}\right)} d t, \\
P_{\mathrm{FA}} & =\int_{\gamma}^{\infty} t^{a_{H 0}-1} \frac{e^{-\frac{t}{b_{H 0}}}}{b_{H 0}^{a_{H 0}} \Gamma\left(a_{H 0}\right)} d t,
\end{aligned}
$$

where $a_{H 0}$ and $b_{H 0}$ are the parameters of the gamma density for null hypothesis $\left(\mathcal{H}_{0}\right)$ and $a_{H 1}$ and $b_{H 1}$ are the parameters of the gamma density for alternate hypothesis $\left(\mathcal{H}_{1}\right)$. It is known that

$$
P_{\mathrm{FA}}=\operatorname{Pr}\left(T(\boldsymbol{Y})>\gamma \mid \mathcal{H}_{0}\right) .
$$

For a given value of $P_{\mathrm{FA}}$, the threshold $\gamma$ is calculated using (49), and the probability of detection is calculated using (48) with the functions available in MATLAB.

\section{Numerical example}

This section provides numerical examples to illustrate the performance of MIMO radar waveform with combined peak and sum power constraints. A MIMO radar system with $M=5$ transmit and $N=5$ receive antenna system is considered. First, we consider the power allocation among the transmit antennas. Figure 1 illustrates the optimal transmitting power on one of the antennas for 100 different target impulse response realizations for various values of the norm, $p$ :

1. $p=1, S P C$ : This case corresponds to the waterfilling strategy, and power is allotted in proportion to the quality of the target mode. More power is allotted to a better mode. For low values of total power, no power is allotted to poor quality modes. As shown in Figure 1, the transmit power fluctuates as much as $4 \mathrm{~W}$ in the transmit antenna under the sum power constraint.

2. $1<p<\infty$, peak and sum power constraints (PSPC): When the value of $p$ is appropriately chosen, this satisfies the sum power constraint of the whole system and the peak power constraint of the individual antenna. If the individual power

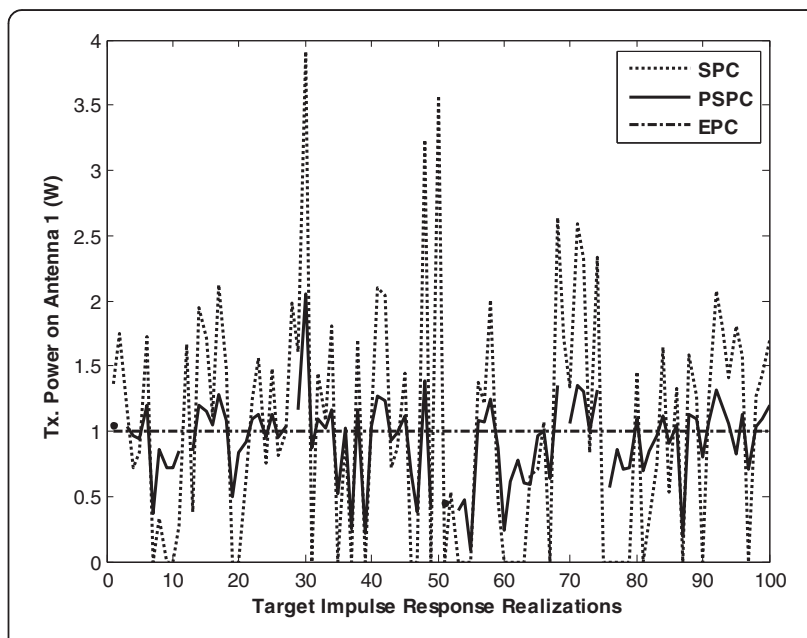

Figure 1 Transmit power across the first antenna. 
constraint is chosen as $2 \mathrm{~W}$, the value of $p$ that satisfies both the sum power constraint and the individual power constraint according to (9) is 2.32 . It is observed in Figure 1 that for the same total power of $5 \mathrm{~W}$, the constraint on the power of the individual antenna has made the power to be distributed to all the five antennas. It is also ensured that the peak power through the individual antenna does not exceed $2 \mathrm{~W}$. If the initial value of the outer iteration $\mu_{0}$ and the initial values for computing $q_{i}^{-1}\left(\mu^{(0)}\right)$ are appropriately chosen, the numerical algorithm yields excellent convergence results with eight digit accuracy after four to six iterations.

3. $p=\infty$, equal power constraint (EPC): For this case, the total power is equally divided among all transmit antennas.

\subsection{Mutual information and MMSE performance}

The MI performance of the three power allocations is shown in Figure 2a,b, and the MMSE performance is

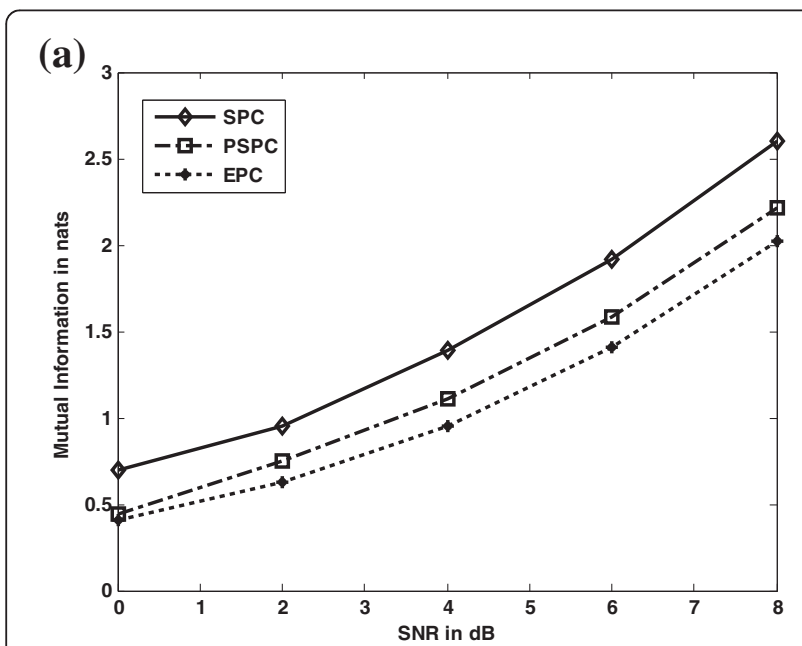

(b)

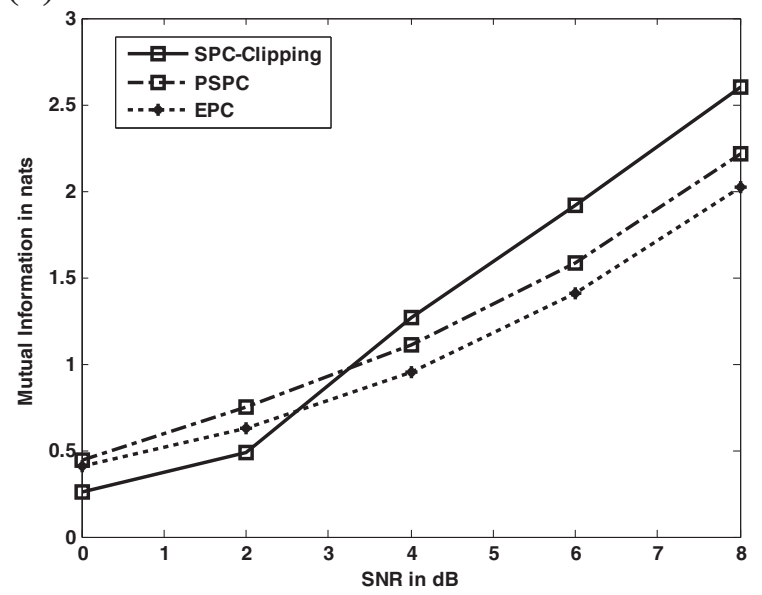

Figure $2 \mathrm{Ml}$ performance (a) without and (b) with constraint on the power amplifier for $c=0.1$. shown in Figure 3a,b. The MI and MMSE values are averaged for 100 different target impulse response realizations.

Figures $2 \mathrm{a}$ and $3 \mathrm{a}$ show the MI performance and MMSE performance, respectively, when there is no power constraint on the power amplifiers used in the transmit antennas. It is observed that the sum power constraint that results in waterfilling power allocation has the best performance. This is analytically attractive, but such a sum power constraint is often unrealistic in practice because in practical implementations each antenna is equipped with its own power amplifier and is limited individually by the linearity of the amplifier. So there would be a maximum limit on the power that could be amplified. The remaining power would be clipped off. If such practical considerations are taken into account, the MI and MMSE performance would be as shown in Figures $2 \mathrm{~b}$ and $3 \mathrm{~b}$. At low signal-to-noise ratio (SNR), the waterfilling power allocation strategy

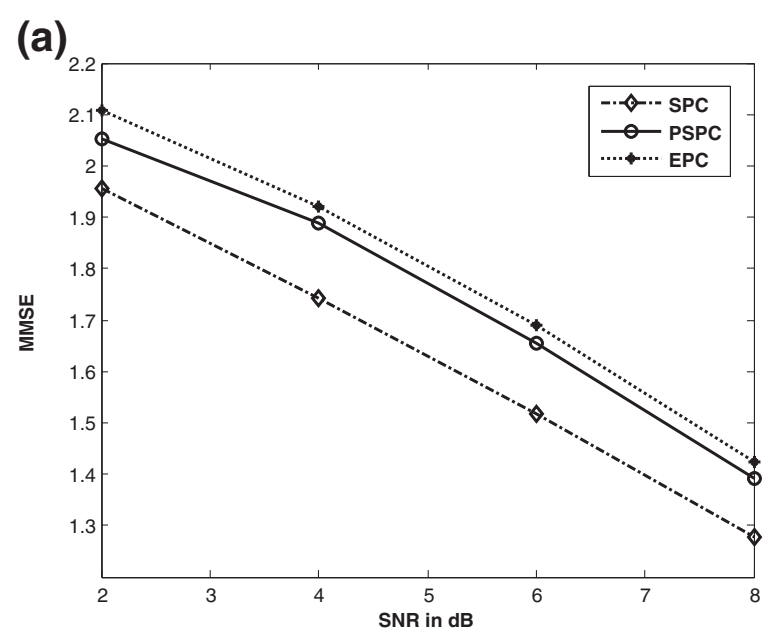

(b)

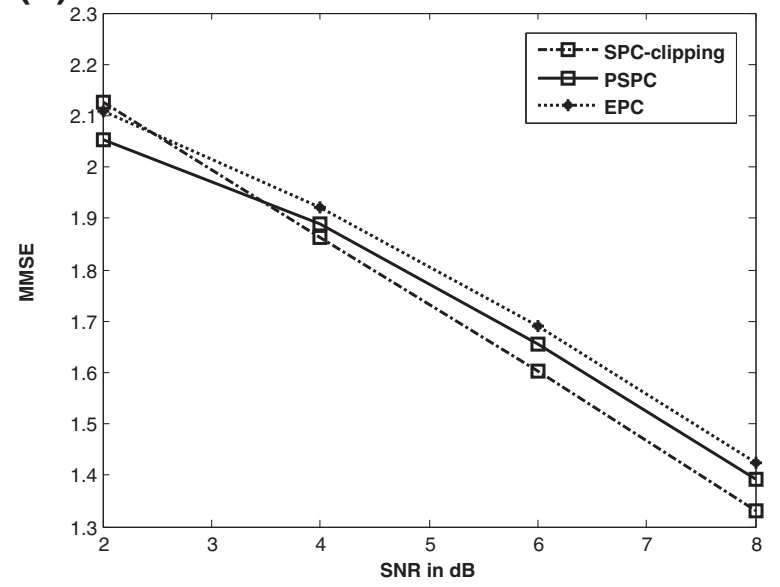

Figure 3 MMSE performance (a) without and (b) with constraint on the power amplifier for $c=0.1$. 
allocates more power to target modes with high target PSD values and no power to some target modes with very low target PSD values. In such circumstances, the power amplifiers would experience a clipping effect and only low power would be transmitted. As given in the problem formulation, the peak power constraint $\alpha$ should be $\beta / M \leq \alpha \leq \beta$. So

$$
\alpha=\frac{\beta}{M}+c \times \beta \text {. }
$$

If $c=0$, then $\alpha=\frac{\beta}{M}$ (equal power), and if $c=1-\frac{1}{M}$, then $\alpha=\beta$ (sum power). So $0 \leq c \leq\left(1-\frac{1}{M}\right)$. In Figures 2 and 3 , it is assumed that $c=0.1$, that is the maximum power that can be transmitted through the power amplifiers is the equal power plus $10 \%$ of the total power (e.g., if $\beta=5 \mathrm{~W}$ for $M=5$, the maximum individual power

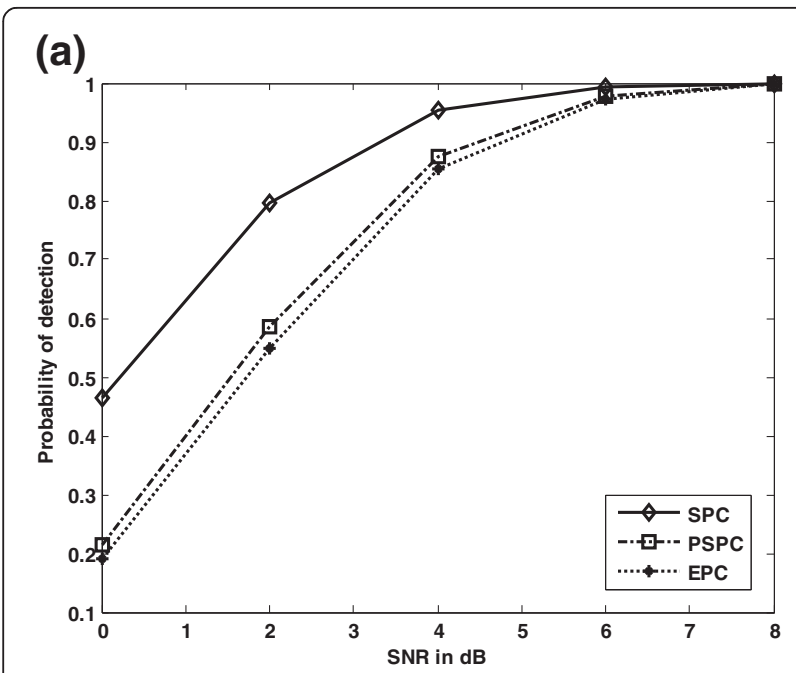

(b)

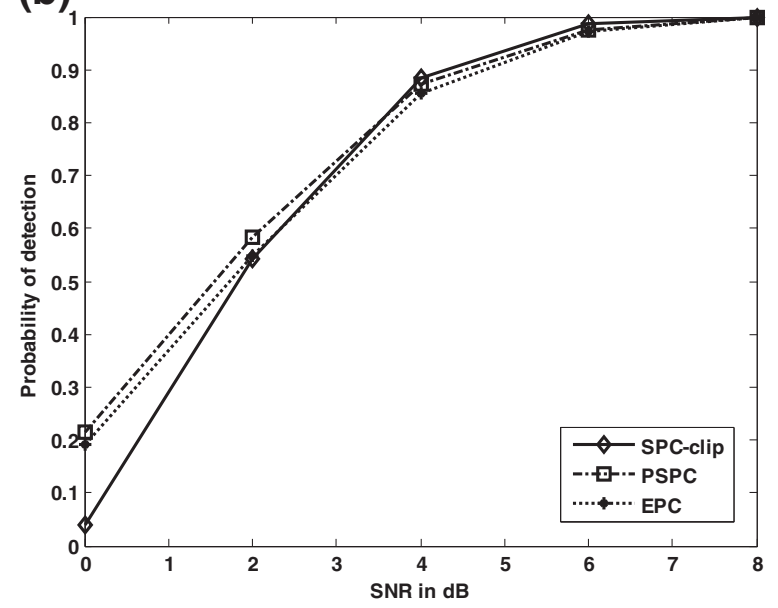

Figure 4 Detection performance of Ml criterion (a) without and (b) with constraint on the power amplifier for $c=0.1$. constraint is $\alpha=1.5 \mathrm{~W})$. It is quite evident that the sum power constraint has got inferior performance up to about an SNR value of $3 \mathrm{~dB}$.

\subsection{Detection performance}

We consider the detection performance of the waveform under the sum power constraint and maximum individual power constraint. It is assumed that the target PSD is known to the transmitter and receiver. The detection performance of the optimal Neyman-Pearson detector is considered. The probability of false alarm is kept as $\operatorname{Pr}_{\mathrm{FA}}=10^{-5}$. To obtain the threshold of the detection statistics and the detection probability, $10^{3}$ Monte Carlo trials are conducted for 100 different target impulse response realizations. The detection performance is shown in Figures 4 and 5 for the MI criterion and Figures 6 and 7 for the MMSE criterion.

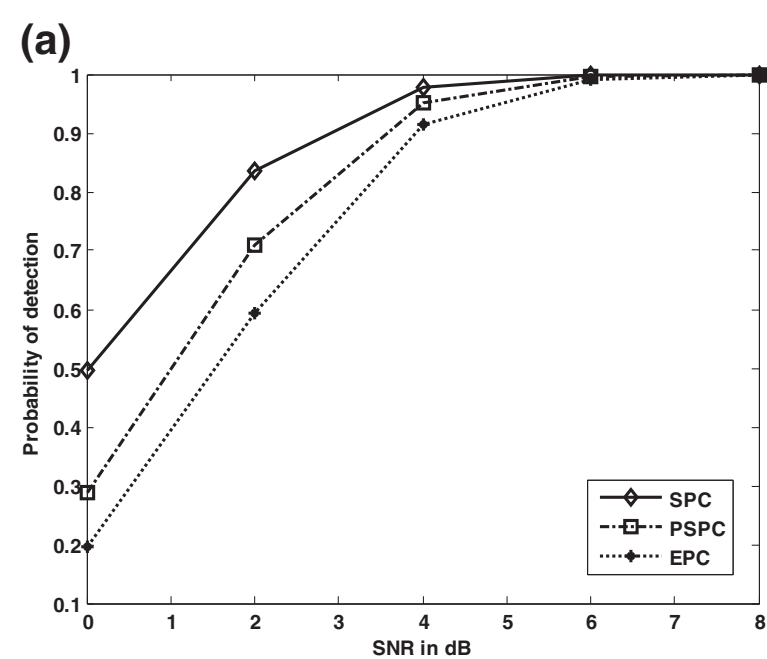

(b)

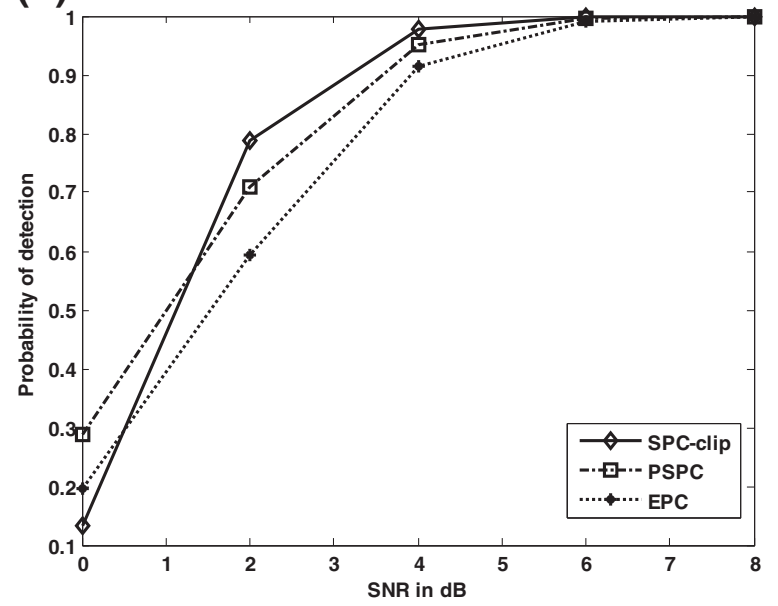

Figure 5 Detection performance of Ml criterion (a) without and (b) with constraint on the power amplifier for $c=0.3$. 
It is observed in Figures 4a and 6a that the detection performance for the sum power constraint that results in the waterfilling type of power allocation is superior compared to that for the peak power constraint and equal power allocation schemes with $c=0.1$ for MI and MMSE criteria, respectively. However, when there is a limitation on the maximum power of the power amplifier in each antenna, the clipping effect would result in inferior detection performance for the waterfilling type of power allocation in a low-SNR region as shown in Figures $4 \mathrm{~b}$ and $6 \mathrm{~b}$. A similar observation is made in Figure $5 \mathrm{a}, \mathrm{b}$ for the MI criterion and Figure $7 \mathrm{a}, \mathrm{b}$ for the MMSE criterion with $c=0.3$ The detection performance of the waveform with equal power allocation in all the antennas is inferior.

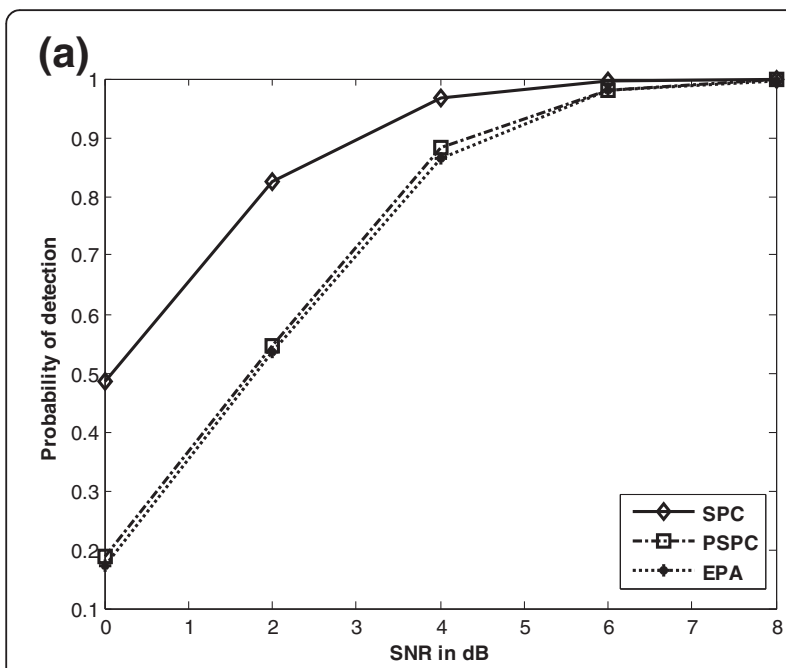

(b)

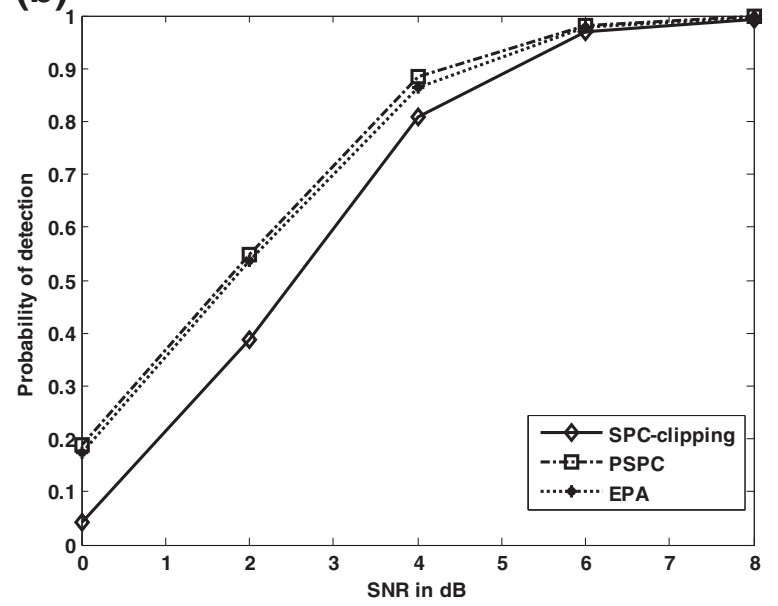

Figure 6 Detection performance of MMSE criterion (a) without and (b) with constraint on the power amplifier for $c=0.1$.

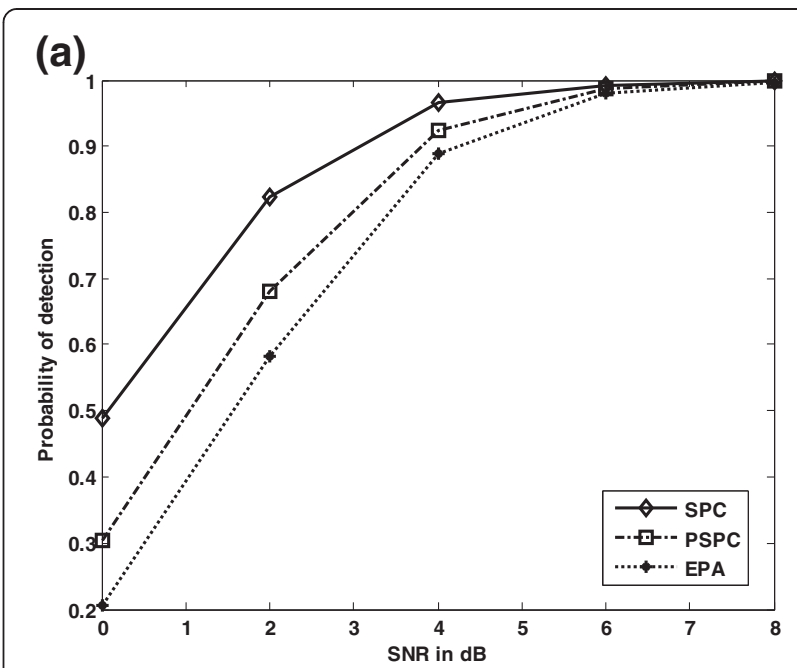

(b)

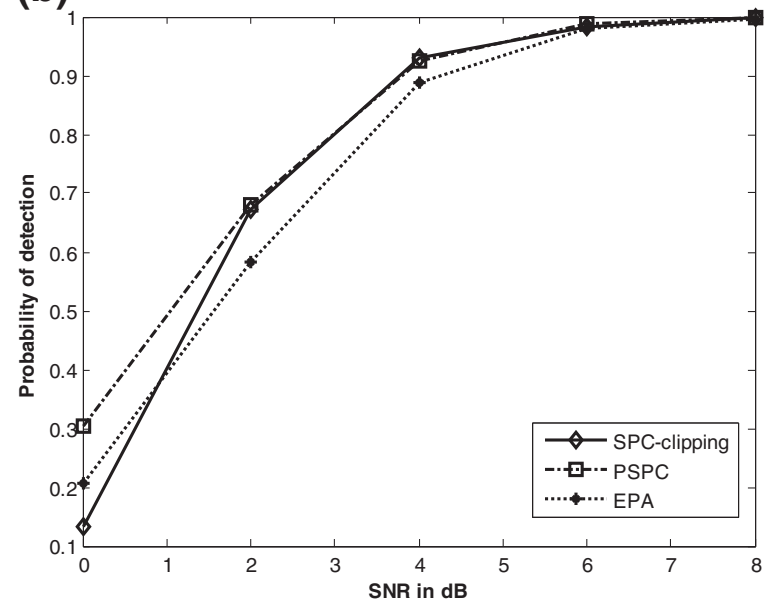

Figure 7 Detection performance of MMSE criterion (a) without and (b) with constraint on the power amplifier for $c=0.3$.

When observing Figures $4 \mathrm{a}$ and $5 \mathrm{a}$ for the MI criterion and Figures $6 a$ and $7 a$ for the MMSE criterion, it is inferred that when the value of individual power constraint $\alpha$ is increased (from $c=0.1$ to $c=$ 0.3 ), the detection performance of the combined peak and sum power constraints move towards the waterfilling case.

\subsection{Convergence behavior}

The combined peak and sum power constraints usea nested Newton algorithm for power allocation. When the initial values are appropriately chosen, the algorithm converges in approximately five to six iterations as shown in Figure 8. 


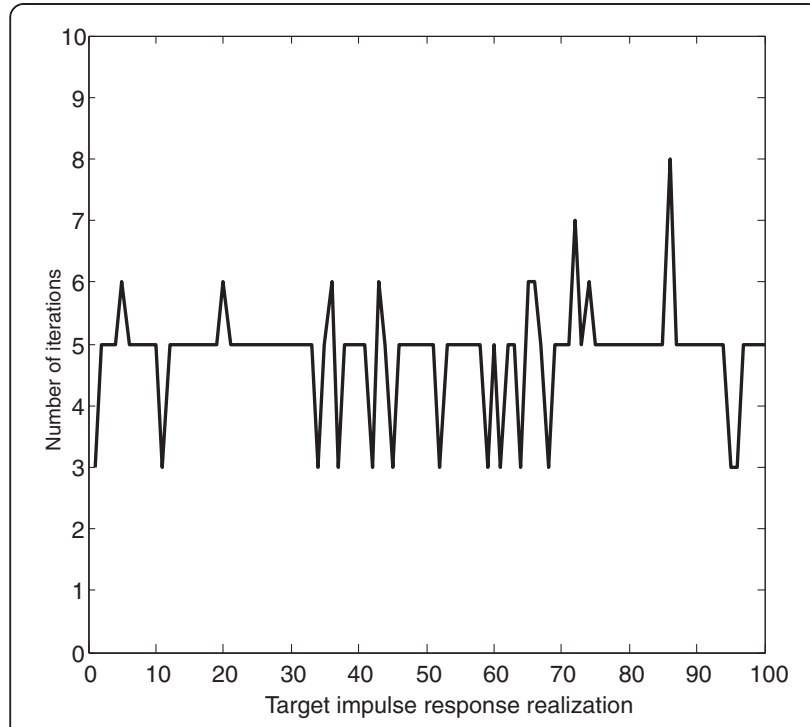

Figure 8 Convergence behavior of the nested Newton algorithm.

\section{Conclusions}

We have investigated the performance of MIMO radar waveform design under peak and sum power constraints. For the design of optimum radar waveform for MIMO radar, the second-order statistics of the extended target impulse response which contains important information regarding the target characteristics is exploited. The KKT approach is used to identify optimal solutions. The well-known waterfilling principle turns out as a special limiting case when $p=1$. For the general case of $1<p<\infty$, it is shown that the power allocation can be determined numerically. The MI performance, MMSE performance, and detection performance are studied for the sum power constraint and peak power constraint. It is observed that in practical implementation when the linearity of the power amplifier is considered, the performance with combined peak and sum power constraints is superior at low SNR. Though the results are suboptimal, this constraint has practical significance. So it is suggested that a hybrid power allocation can be adopted. At low SNR, combined peak and sum power constraints can be used, and at high SNR, the sum power constraint can be used.

\section{Abbreviations}

EPC: Equal power constraint; KKT: Karush-Kuhn-Tucker; MI: Mutual information; MIMO: Multiple-input multiple-output; MMSE: Minimum mean square error; PSPC: Peak and sum power constraints; PSD: Power spectral density; pdf: Probability density function; SPC: Sum power constraint.

\section{Competing interests}

The authors declare that they have no competing interests.

\section{Author details}

${ }^{1}$ Department of Electronics and Communication Engineering, Sethu Institute of Technology, Kariapatti, Virudhunagar District 626 115, India. ${ }^{2}$ TIFAC-CORE in Wireless Technologies, Department of Electronics and Communication Engineering, Thiagarajar College of Engineering, Madurai 625 015, India.
Received: 19 April 2013 Accepted: 11 June 2013

Published: 7 July 2013

\section{References}

1. PM Woodward, IL Davies, A theory of radar information. Philos. Mag. 41, 1001-1017 (1950)

2. PM Woodward, Information theory and the design of radar receivers. Proc. IRE 39, 1521-1524 (1951)

3. MR Bell, Information theory and radar waveform design. IEEE Trans. Inf. Theory 39, 1578-1597 (1993)

4. A Leshem, A Nehorai, Information theoretic radar waveform design for multiple targets, in Proceedings of Conference on Information Sciences and Systems (CISS) (Princeton, 2006), pp. 1408-1412

5. JR Roman, JW Garnham, P Antonik, Information theoretic criterion for waveform selection, in Proceedings of IEEE Workshop on Sensor Array and Multichannel Processing (SAM) (Waltham, 2006), pp. 444-448

6. Y Yang, RS Blum, MIMO radar waveform design based on mutual information and minimum mean-square error estimation. IEEE Trans. Aerosp. Electron. Syst. 43, 330-343 (2007)

7. E Fishler, A Haimovich, RS Blum, D Chizhik, L Cimini, R Valenzuela, Spatial diversity in radars - models and detection performance. IEEE Trans. Signal Process. 54, 823-838 (2006)

8. E Fishler, A Haimovich, RS Blum, D Chizhik, L Cimini, R Valenzuela, Statistical MIMO radar, in Proceedings of the 12th Annual Workshop on Adaptive Sensor Array Processing (Lexington, 2003)

9. E Fishler, A Haimovich, RS Blum, D Chizhik, L Cimini, R Valenzuela, MIMO radar: an idea whose time has come, in Proceedings of 2004 IEEE Radar Conference (Philadelphia, 2004)

10. E Fishler, A Haimovich, RS Blum, L Cimini, D Chizhik, R Valenzuela, Performance of $\mathrm{MIMO}$ radar systems: advantages of angular diversity, in Proceedings of the 38th Asilomar Conference on Signals, Systems and Computers (Pacific Grove, 2004)

11. DR Fuhrmann, G San Antonio, Transmit beamforming for MIMO radar systems using partial signal correlation, in Proceedings of the 38th Asilomar Conference on Signals, Systems and Computers (Pacific Grove, 2004)

12. F Robey, S Coutts, D Weikle, J McHarg, K Cuomo, MIMO radar theory and experimental results, in Proceedings of the 38th Asilomar Conference on Signals, Systems and Computers (Pacific Grove, 2004)

13. K Forsythe, D Bliss, G Fawcett, Multiple-input multiple-output (MIMO) radar: performance issues, in Proceedings of the 38th Asilomar Conference on Signals, Systems and Computers (Pacific Grove, 2004)

14. DJ Bliss, K Forsythe, Multiple-input multiple-output (MIMO) radar and imaging: degrees of freedom and resolution, in Proceedings of the 37th Asilomar Conference on Signals, Systems and Computers (Pacific Grove, 2003)

15. D Rabideau, P Parker, Ubiquitous MIMO digital array radar, in Proceedings of the 37th Asilomar Conference on Signals, Systems and Computers (Pacific Grove, 2003)

16. SA Kassam, HV Poor, Robust techniques for signal processing: a survey. Proc. IEEE 73(3), 433-481 (1985)

17. JH Kotecha, AM Sayeed, Transmit signal design for optimal estimation of correlated MIMO channels. IEEE Trans. Signal Process. 52, 546-557 (2004)

18. T Naghibi, F Behnia, MMSE based waveform design for MIMO radars, in Proceedings of IET Conference on Waveform Diversity \& Digital Radar (London, 2008), pp. 1-5

19. Z Wenshu, Y Liuqing, Communications-inspired sensing: a case study on waveform design. IEEE Trans. Signal Process. 58, 2 (2010)

20. A Merline, SJ Thiruvengadam, Proceedings of International Conference on Signal Processing and Communication (SPCOM), in Proceedings of International Conference on Signal Processing and Communication (SPCOM) (Bangalore, 2012), pp. 1-5

21. $X$ Zhang, Y Xie, J Li, P Stoica, MIMO transmit beamforming under uniform elemental power constraint. IEEE Trans. Signal Process. 55(11), 5395-5406 (2007)

22. DP Palomar, Unified framework for linear MIMO transceivers with shaping constraints. IEEE Commun. Lett. 8(12), 697-699 (2004)

23. Y Wei, T Lan, Transmitter optimization for the multi-antenna downlink with per-antenna power constraint. IEEE Trans. Signal Process. 55, 2646-2660 (2007)

24. J Dai, Z Ye, Optimal designs for linear MIMO transceivers using directional derivative. IET Commun. 3, 1452-1462 (2009) 
25. A Feiten, R Mathar, S Hanly, Eigenvalue-based optimum-power allocation for Gaussian vector channels. IEEE Trans. Inform. Theory 53, 2304-2309 (2007)

26. TM Cover, JA Thomas, Elements of Information Theory (Wiley, New York 1991)

27. TM Cover, A Gamal, An information-theoretic proof of Hadamard's inequality. IEEE Trans. Info. Theory 29, 930-931 (1983)

28. S Boyd, L Vandenberghe, Convex Optimization (Cambridge University Press, New York, 2004)

29. GB Giannakis, S Zhou, Optimal transmit-diversity precoders for random fading channels, in Proceedings of the IEEE Global Telecommunications Conference (San Francisco, 2000), pp. 1839-1843

30. T Bo, T Jun, $\mathrm{P}$ Yingning, MIMO radar waveform design in colored noise based on information theory. IEEE Trans. Signal Process. 58(9), 4684-4697 (2010)

31. TW Anderson, An Introduction to Multivariate Statistical Analysis, 3rd edn. (Wiley, New York, 2003)

32. AH Feiveson, FC Delaney, The Distribution and Properties of a Weighted Sum of Chi Squares. NASA Technical Note (NASA, Springfield, 1968)

doi:10.1186/1687-6180-2013-127

Cite this article as: Arulraj and Jeyaraman: MIMO radar waveform design with peak and sum power constraints. EURASIP Journal on Advances in Signal Processing 2013 2013:127

Submit your manuscript to a SpringerOpen ${ }^{\circ}$ journal and benefit from:

- Convenient online submission

- Rigorous peer review

- Immediate publication on acceptance

- Open access: articles freely available online

- High visibility within the field

- Retaining the copyright to your article

Submit your next manuscript at $>$ springeropen.com 